Open Access

\title{
The formation and design of the TRIAGE study - baseline data on 6005 consecutive patients admitted to hospital from the emergency department
}

Louis Lind Plesner ${ }^{1,2^{*}}$, Anne Kristine Servais Iversen ${ }^{1,2}$, Sandra Langkjær ${ }^{1,2}$, Ture Lange Nielsen ${ }^{1,2}$, Rebecca Østervig ${ }^{1,2}$, Peder Emil Warming ${ }^{1,2}$, Idrees Ahmad Salam ${ }^{1,2}$, Michael Kristensen ${ }^{1,2}$, Morten Schou ${ }^{1,2}$, Jesper Eugen-Olsen ${ }^{3}$, Jakob Lundager Forberg ${ }^{4}$, Lars Køber ${ }^{5}$, Lars S. Rasmussen ${ }^{6}$, György Sölétormos ${ }^{7}$, Bente Klarlund Pedersen ${ }^{8}$ and Kasper Iversen ${ }^{1,2}$

\begin{abstract}
Background: Patient crowding in emergency departments (ED) is a common challenge and associated with worsened outcome for the patients. Previous studies on biomarkers in the ED setting has focused on identification of high risk patients, and and the ability to use biomarkers to identify low-risk patients has only been sparsely examined. The broader aims of the TRIAGE study are to develop methods to identify low-risk patients appropriate for early ED discharge by combining information from a wide range of new inflammatory biomarkers and vital signs, the present baseline article aims to describe the formation of the TRIAGE database and characteristize the included patients.
\end{abstract}

Methods: We included consecutive patients $\geq 17$ years admitted to hospital after triage staging in the ED. Blood samples for a biobank were collected and plasma stored in a freezer $\left(-80^{\circ} \mathrm{C}\right)$. Triage was done by a trained nurse using the Danish Emergency Proces Triage (DEPT) which categorizes patients as green (not urgent), yellow (urgent), orange (emergent) or red (rescusitation). Presenting complaints, admission diagnoses, comorbidities, length of stay, and 'events' during admission (any of 20 predefined definitive treatments that necessitates in-hospital care), vital signs and routine laboratory tests taken in the ED were aslo included in the database.

Results: Between September $5^{\text {th }} 2013$ and December $6^{\text {th }} 2013,6005$ patients were included in the database and the biobank (94.1\% of all admissions). Of these, 1978 (32.9\%) were categorized as green, 2386 (39.7 \%) yellow, 1616 (26.9\%) orange and 25 (0.4\%) red. Median age was 62 years (IQR 46-76), $49.8 \%$ were male and median length of stay was 1 day (IQR 0-4). No events were found in 2658 (44.2\%) and 158 (2.6\%) were admitted to intensive or intermediate-intensive care unit and 219 (3.6\%) died within 30 days. A higher triage acuity level was associated with numerous events, including acute surgery, endovascular intervention, i.v. treatment, cardiac arrest, stroke, admission to intensive care, hospital transfer, and mortality within 30 days $(p<0.001)$.

(Continued on next page)

\footnotetext{
* Correspondence: louislindplesner@gmail.com

'Department of Cardiology, Endocrinology and Nephrology, North Zealand

Hospital, Copenhagen University Hospital, Copenhagen, Denmark

2Department of Cardiology, Herlev Hospital, Copenhagen University Hospital,

Copenhagen, Denmark

Full list of author information is available at the end of the article
}

(c) 2015 Plesner et al. Open Access This article is distributed under the terms of the Creative Commons Attribution 4.0 International License (http://creativecommons.org/licenses/by/4.0/), which permits unrestricted use, distribution, and reproduction in any medium, provided you give appropriate credit to the original author(s) and the source, provide a link to the Creative Commons license, and indicate if changes were made. The Creative Commons Public Domain Dedication waiver (http://creativecommons.org/publicdomain/zero/1.0/) applies to the data made available in this article, unless otherwise stated. 


\section{(Continued from previous page) \\ Conclusion: The TRIAGE database has been completed and includes data and blood samples from 6005 unselected consecutive hospitalized patients. More than $40 \%$ experienced no events and were therefore potentially unnecessary hospital admissions.}

Keywords: Triage, Biomarkers, Emergency department risk stratification, Avoidable hospitalization, Biobank

\section{Background}

There are annually around 800.000 acute admissions of adults in Denmark, and numbers have increased in recent years [1]. Crowding in emergency departments (ED) and hospital wards are common problems and can be detrimental to patient outcome [2-4]. It has recently been estimated that over $20 \%$ of all acute hospital admissions are potentially avoidable [5]. Discovering ways of reducing unnecessary hospitalization is essential, as hospital admission is associated with adverse events such as infections, accidental injuries and cardiovascular complications [6], and early discharge has been shown to improve outcome [7, 8].

Triage algorithms for stratifying patients in the ED according to acuity level have been developed and employed for the purpose of prioritizing ressources and ensuring adequate attention to the sickest patients [9]. The present triage algorithms have not been designed to identify patients in the ED with such a low need of acute treatment, that they can be immediately discharged to an outpatient clinic or follow-up by their own general practitioner.

New efficient tools enabling identification of these patients could result in substantial economical savings [10], provide better time to treat the high-risk patients and probably reduce adverse events and inconvenience associated with unnecessary hospitalizations of low-risk patients.

Several old and new biomarkers are useful to estimate short term prognosis [11-16] and a recent theoretical study has suggested that the use of biomarkers would substantially strengthen the existing triage algorithms [17].

The TRIAGE study is designed primarily to examine if a system combining biochemical markers with currently available data, including observations by a triage nurse, can identify patients who can be safely discharged to outpatient follow-up. Secondary objectives of the TRIAGE study are to investigate the prognostic value of several new and old biomarkers in a large population of unselected and consecutively admitted patients. These primary and secondary aims will be assesed in future publications.

The aims of the present article are to describe the formation of the TRIAGE database, the design of the study, and the characteristics of the included patients.

\section{Methods/Design}

The formation of the database for the TRIAGE study took place at North Zealand University Hospital, which is a large hospital in the Capital Region of Denmark. The hospital is a 24-h acute care hospital offering medical, surgical, level-2 trauma, emergency and intensive care services for 310000 citizens in North Zealand [18].

\section{Study design}

The TRIAGE study was prospective and observational. There was non-stop inclusion of consecutive patients presenting to an emergency department (24 h 7 days/week) until target number was met $(=6000)$. Admission, and thereby inclusion in the study, was defined as refferal to a bed and blood samples drawn in the ED. We included all patients admitted through the emergency department, but patients $\leq 17$ years and obstetric patients were not included since they were admitted directly to the paediatric ED or obstetrics department, respectively. Patients detected in the field with major trauma, ST-elevation acute myocardial infarction or stroke within $2-3 \mathrm{~h}$ were admitted to the tertiary centre in the region.

\section{Data collection}

All data were merged using the unique personal CPR number ('Central Personal Registry') into a secure webbased database, except the biomarker results. The biomarkers will be registered in an additional database (blinded and keyed by a subject identifyer). After the databases have been completed, all data will be merged and transferred to a central public server in Statistics Denmark and blinded prior to further analyses. Ongoing information about death, hospitalization and medication can be retreived from these central servers. The data derive from the three different sources listed below.

\section{Triage data}

Data were obtained by trained and experienced triagenurses at the time of admission and entered into the database by a medical student and included age, gender, vital signs and triage category. Table 1 shows the five-level $\mathrm{Da}$ nish Emergency Proces Triage (DEPT) used in the ED, patients are categorised into five triage levels based on vital signs and a presenting complaint algorithm [19]. DEPT is a Danish adaption and modification of the "Adaptive Process Triage" (ADAPT) developed in Sweden in 2006 [20]. Patients triaged blue were not included in the study as they were never admitted at North Zealand Hospital according to our definition. Heart rate (HR), blood pressure $(\mathrm{BP})$, respiratory rate $(\mathrm{RR})$ and peripheral arterial oxygen 
Table 1 The five categories of DEPT triage

\begin{tabular}{llll}
\hline Rescuscitation & Emergent & Urgent & Non-urgent \\
\hline Life threatened & Critical illness & Potentially unstable & Stable \\
Constant re-evaluation & Re-evaluation every $15 \mathrm{~min}$. & Re-evaluation every 60 min. & Re-evaluation every 180 min. $\quad$ Re-evaluation every 240 min. \\
Acute Team & Physician and Nurse & Nurse $\rightarrow$ physician* & Nurse $\rightarrow$ Physician* \\
\hline
\end{tabular}

DEPT (Danish Emergency Process) Triage: Each patient is assigned a triage level for each of the two main descriptors: 1) Vital signs and 2) presenting complaint. The nurse performing triage can increase a patients triage level if she believes the patient is more ill than what is determined by DEPT [19]. *Patients are seen by a physician, but the evaluation can be made by a nurse initially

saturation $\left(\mathrm{SpO}_{2}\right)$ were measured on a patient monitor (Phillips Intellivue MP30) and temperature (Tp) was measured with an ear thermometer (Covidien Genius ${ }^{\mathrm{TM}} 2$ ).

\section{Blood sample data}

Blood samples were drawn by either a phlebotomist or a medical student as soon as possible (within 0-60 min following admission) and the procedure followed a predefined sequence to minimize the risk of preanalytical errors [21].

Results from routine laboratory examinations were later retrieved from the hospital database (LABKA ll. Version 2.5.0.H2 Computer Sciences Corporation (CSC)) including CRP, hemoglobin, sodium, potassium, creatinine, albumin (Dimension Vista ${ }^{\circ}$ 1500, Siemens Medical Solutions Diagnostics), and white blood cell count as well as platelet count (Sysmex XE-5000, Sysmex Corporation).

Additional blood was collected for storage in the biobank and subsequent analysis of a wide range of new inflammatory biomarkers. Blood samples were drawn simultaneously with the routine laboratory tests, and they were spun for $10 \mathrm{~min}$ at $1800 \mathrm{G}$ within $120 \mathrm{~min}$ and transferred to 13 different cryotubes ( 8 serum, 4 plasma and 1 buffycoat per patient), each tube containing 0.5-1 ml. The cryotubes were immediately frozen at $-20{ }^{\circ} \mathrm{C}$ and transferred to a central $-80{ }^{\circ} \mathrm{C}$ freezer on the following day for final storage. The laboratory procedures were performed by medical students, who had been carefully instructed prior to the study.

\section{Data from the patient file}

Data on comorbidity, smoking habits, alcohol intake, social status, events during hospitalization (any of 20 predefined definitive treatments that necessitates inhospital care, i.e., Acute surgery, endovascular procedure, death within 30 days etc. - see Table 3. The events had been predefined in the study protocol), hospital length of stay (LOS), and hospital discharge diagnosis were also retrieved from the patient file (OPUS Arbejdsplads, version 2.5.0.0 Computer Sciences Corporation (CSC)). Information about death within 30 days after admission was retrieved from the central public server in Statistics Denmark.

\section{Identification of unnecessary admissions}

An unnecessary hospitalization was defined as a situation where a patient could have been handled by the general practioner or by an outpatient clinic within 1-2 weeks. Patients who experienced at least one of the predefined events, were identified and categorized as definate necessary hospitalizations, but the list of events was not constructed to deem whether a hospitalization could have been unnessesary as it has previously been argued that a list of predefined criteria for measuring the appropriateness of an admission can never be sufficient [22]. Therefore we had two specialists in internal medicine or emergency medicine independently assessing the files of patients with none of the specified events to determine if it could be considered an unnecessary or necessary admission. Only when both specialists agreed, the admission was considered unnecessary. The specialists were blinded from the information of whether they were the primary or secondary reviewer of the patient case and from the judgement of the other.

\section{Identification of high-risk patients}

Patients with admission to intensive care unit within 30 days or semi-intensive care unit within 14 days or death within 30 days will be categorized as high-risk patients.

\section{Ethics}

The study was conducted according to Danish ethical regulations and was approved by the Danish Data Protection agency (J. 2007-58-0015).

\section{Consent}

Regarding consent from patients, formal ethical approval was not necessary for this study. After completion of the biobank, all data will be transferred to a central server. All patients will thereafter be blinded, but with a unique patient number. It is thereafter possible to connect the triage database with all other Danish registers and get information of death, biochemistry, medication, admissions etc. This procedure is in accordance with ethical regulations.

\section{Sample size}

A definite sample size was not caluclated prior to the study, as the percentage of patients with unnecessary 
admission were unknown. We anticipated that at least 20 $\%$ of the population would be unnecessarily admitted. A triage used for sending patients home directly from the ED should ensure that the risk of it being a necessary admission should be very small (less than $0.1 \%$ corresponding to a specificity of $99.9 \%$ ). The sensitivity of the model should be at least $20 \%$. Thus, with 6000 admissions the triage should be able to identify 240 (20 \%) of the hypothesized 1200 unnecessary admissions, that could be discharged immediately.

\section{Statistics}

Continuous data are presented as mean with standard deviation (SD) or as median with interquartile range (IQR) where appropriate and categorical data are presented as $\mathrm{n}(\%)$. Continuous variables are compared using the Student's $t$-test and categorical variables are compared using the Chi-square-test. One-way ANOVA or Kruskal Wallis $\mathrm{H}$ test are used to compare continuous data across the 4 triage categories when data is normally or non-normally distributed, respectively. Reporting frequencies of events by different investigators were compared using chi-squared tests. were A two-sided probability of $p<0.05$ was considered statistically significant. All analyses were performed using SPSS software version 22.0 (IBM corp., USA).

\section{Results}

We included data on 6005 consecutive patient admissions between September 5th, 2013 and December 6th, 2013. This corresponds to $94.1 \%$ of the total number of admitted patients $(\mathrm{n}=6383)$ in the study period (Fig. 1).

Median age was 62 years (IQR 46-76) and $49.8 \%$ were males. Median LOS was 1 day (IQR 0-4). Mean vital values were: systolic BP $136 \mathrm{mmHg}$ (SD 23.5), HR 82 per min (SD 19), $\mathrm{Tp} 36.8{ }^{\circ} \mathrm{C}$ (SD 0.8), $\mathrm{SpO}_{2} 97.7 \%$ (SD 2.5) and RR 17 per min (SD 3.8). There were 570 patients $(9.5 \%)$ who recieved oxygen therapy during $\mathrm{SpO}_{2}$ measurement. Vital

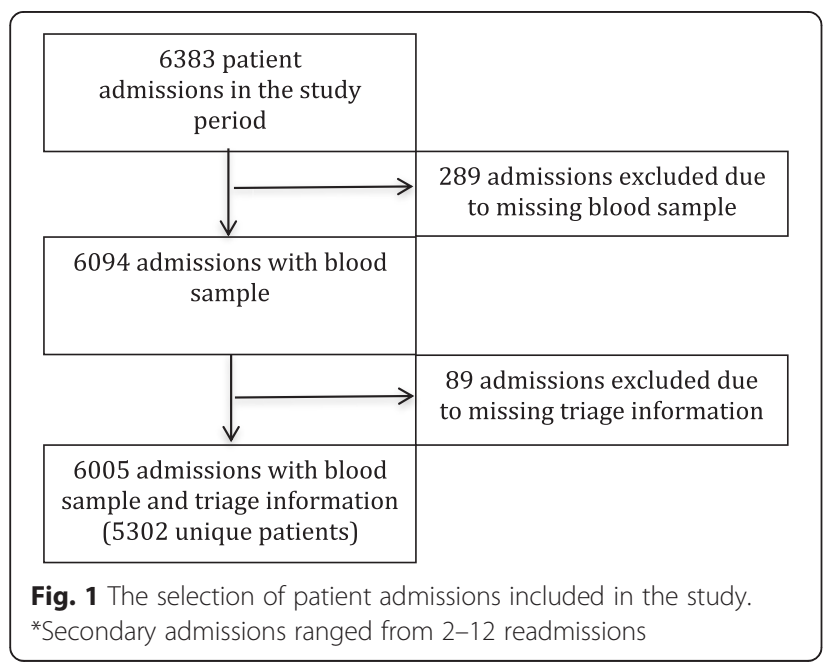

values according to triage stage are shown in Additional file 1: Table S1. A total of 2658 patients (44.2\%) experienced no events during admission, while 158 (2.6\%) were admitted to intensive or intermediate-intensive care unit and 219 (3.6 \%) died within 30 days. Distribution of patient presenting complaints and admission diagnoses are found in Figs. 2 and 3, respectively.

Patient characteristics according to triage category are displayed in Table 2. There was a significant relationship between level of urgency and increasing age, male gender, and comorbidity. There was a significant association between triage category and numerous events during hospitalization, including acute surgery, endovascular intervention, i.v. treatment, cardiac arrest, stroke, admission to intensive care, transfer to another hospital, and mortality within 30 days (Table 3 ).

There was a significant variation in reporting frequency across different investigators for only two events: trombolysis $(0 \%-1.2 \%, p=0.04)$ and admission to intensive care $(0.8-3.6 \%, p<0.01)$.

\section{Characteristics of the patient admissions not included}

There were 378 (5.9\%) patient admissions not included for further analysis, due to missing triage category and/ or blood sample for the biobank. Compared to the included patients, they had a higher age, longer LOS, lower $\mathrm{BP}$ and $\mathrm{SpO}_{2}$ at admission, as well as higher $\mathrm{HR}$ and RR. Significantly more patients were triaged red or orange (35.4 \% vs. $27.3 \%)$. Many events were more common in this group: i.v. treatment, i.v.-diuretics, antibiotic treatment, admissions lasting more than 3 days, not due to social factors or complications due or to initiated treatments or investigations within 30 days, cardiac arrest within 30 days, admission to intensive or semi-intensive care and death within 30 days. They also had a significantly higher burden of comorbidities such as diabetes, kidney disease, liver disease, rheumatic disease, use of alcohol and smoking. They were more often living alone, residents of a nursing home or subjects to domestic help. (All data including $p$-values are presented in Additional file 2: Table S2)

There were no events in 106 (28\%) of the patient admissions not included corresponding to $3.8 \%$ of the total number of admissions with no events.

\section{Missing data}

Presenting complaint or admission diagnosis was lacking in the patient file in $<2 \%$ of cases (Figs. 2 and 3), and all 8 routine laboratory tests were available in 5548 admissions (92.4 \%). BP, HR and $\mathrm{SpO}_{2}$ were all available in $84 \%$ of admissions. The most frequently missing variables from the triage were temperature $(27.4 \%$ missing) and respiratory rate (18.4\% missing) (Additional file 1: Table S1). 


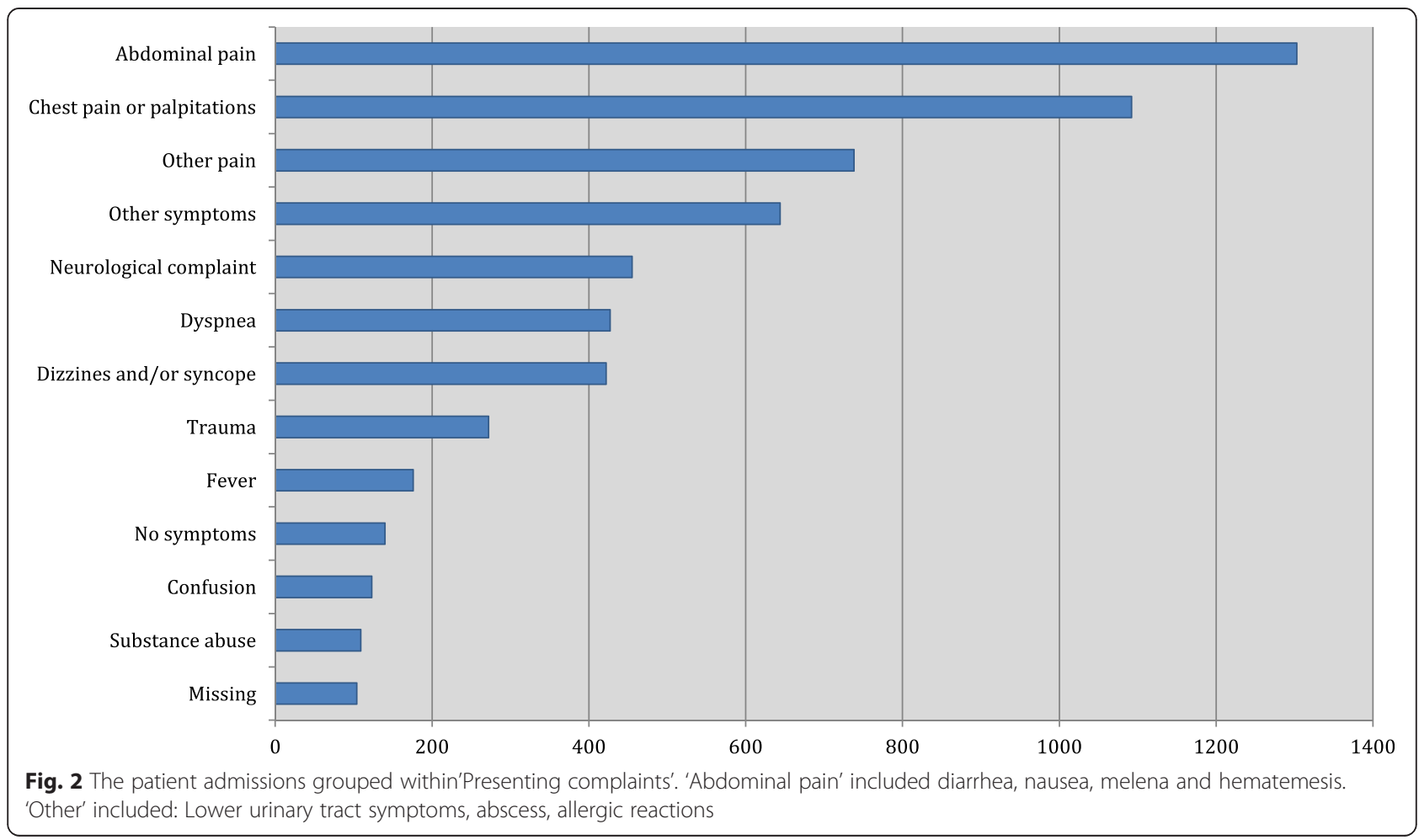

\section{Discussion}

We achieved the goal of assembling a database with data and blood samples from more than 6000 admissions of consecutive patients admitted to the hospital through the emergency department. Patients were included $24 \mathrm{~h}$ a day, 7 days a week, which resulted in inclusion of 94.1 $\%$ of all admitted patients.

Crowding in the emergency department can be detrimental to patient outcome $[2,3]$ and with economical cutbacks in the health sector, prioritizing resources is crucial. A

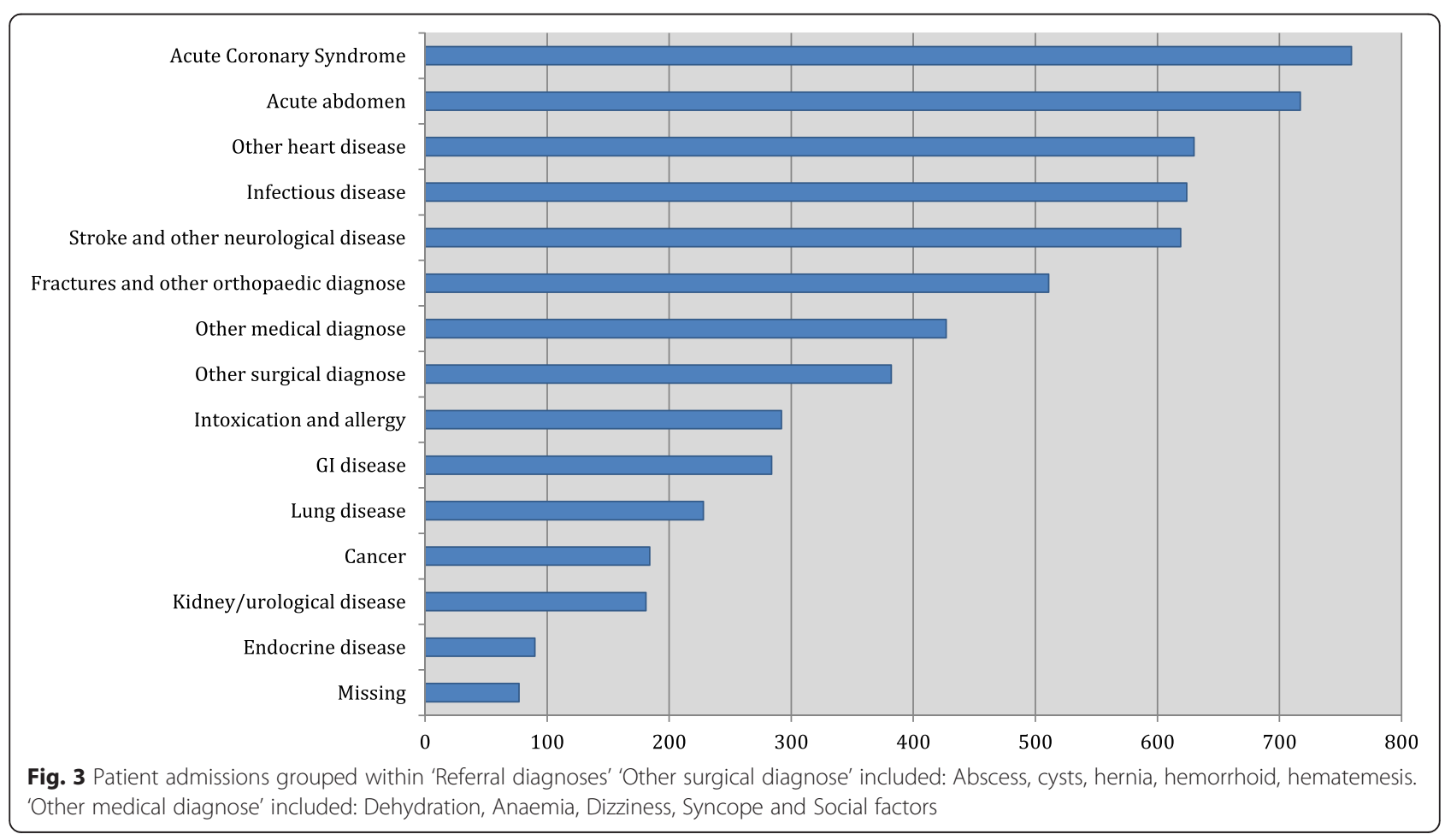


Table 2 Demographics and comorbidities according to Triage stage (unique patients)

\begin{tabular}{|c|c|c|c|c|c|}
\hline & Green $(n=1741)$ & Yellow $(n=2094)$ & Orange $(n=1445)$ & $\operatorname{Red}(n=22)$ & $P$ value* \\
\hline Age, median years (IQR) & $61(44-76)$ & $61(44-76)$ & $64(50-77)$ & $72(66-82)$ & $<0.001$ \\
\hline Gender, male n (\%) & $825(47.4)$ & $1015(48.5)$ & $747(51.7)$ & $17(77.3)$ & 0.004 \\
\hline LOS, median days (IQR) & $1.0(0-3)$ & $1.0(0-4)$ & $1.0(1-5)$ & $5.0(1.75-8)$ & $<0.001$ \\
\hline Ischemic heart disease n (\%) & $109(6.3)$ & $150(7.2)$ & $223(15.4)$ & $4(18.2)$ & $<0.001$ \\
\hline Heart failure, n (\%) & $54(3.1)$ & $102(4.9)$ & $112(7.8)$ & $5(22.7)$ & $<0.001$ \\
\hline Hypertension, n (\%) & $352(20.2)$ & $420(20.1)$ & $400(27.7)$ & $7(31.8)$ & $<0.001$ \\
\hline Diabetes, n (\%) & $171(9.8)$ & $189(9.0)$ & $187(12.9)$ & $5(22.7)$ & $<0.001$ \\
\hline COPD, n (\%) & $96(5.5)$ & $171(8.2)$ & $131(9.1)$ & $4(18.2)$ & $<0.001$ \\
\hline Kidney disease, n (\%) & $56(3.2)$ & $75(3.6)$ & $49(3.4)$ & $1(4.5)$ & 0.925 \\
\hline Liver disease, n (\%) & $29(1.7)$ & $31(1.5)$ & $15(1.0)$ & $2(9.1)$ & 0.010 \\
\hline Rheumatic disease, n (\%) & $34(2.0)$ & $47(2.2)$ & $26(1.8)$ & $0(0.0)$ & 0.709 \\
\hline Cancer, n (\%) & $248(14.2)$ & $292(13.9)$ & $179(12.4)$ & $2(9.1)$ & 0.389 \\
\hline Smoking, n (\%): & & & & & $<0.001$ \\
\hline Former & $314(18.0)$ & $456(21.8)$ & $381(26.4)$ & $5(22.7)$ & \\
\hline Current & $405(23.3)$ & $488(23.3)$ & $368(25.5)$ & $8(36.4)$ & \\
\hline Alcohol abuse, n (\%) & $143(8.2)$ & $156(7.4)$ & $109(7.5)$ & $5(22.7)$ & 0.052 \\
\hline \multicolumn{6}{|l|}{ Social aspects, n (\%): } \\
\hline Living alone & $352(20.2)$ & $396(18.9)$ & $341(23.6)$ & $3(13.6)$ & 0.006 \\
\hline Domestic help & $153(8.8)$ & $160(7.6)$ & $147(10.2)$ & $2(9.1)$ & 0.075 \\
\hline Nursing home & $77(4.4)$ & $107(5.1)$ & $67(4.6)$ & $1(4.5)$ & 0.788 \\
\hline
\end{tabular}

* $P$ value indicates significant difference between the triage categories. Alcohol abuse was defined as $\geq 3$ units of alcohol/day LOS Lenght of stay, COPD Chronic obstructive pulmonary disease. Green/yellow/orange/red refers to triage acuity level in DEPT: non-urgent/urgent/emergent/ resuscitation respectively, (Table 1 for detalis)

recent Danish study based on 2.65 million patient admissions shows that a high bed occupancy significantly increases in-hospital mortality and 30-day mortality [4]. In a recent article by O'Cathain et al., $22 \%$ of almost 15000 000 acute emergency admissions were deemed potentially unnecessary based on review of the admission diagnoses, searching for conditions know to be rich in avoidable admissions [5]. However, early identification of low-risk patients in the emergency department can be challenging. ER crowding could potentially be alleviated by faster discharge and/or faster admission to an inpatient bed.

The use of clinical scores for risk prediction have previously shown benefit primarily in groups of patients and not individuals [23], but even though previous models have not had the diagnostic quality to apply on individuals it is still essential to retest this idea in a more modern and advanced model. Future studies will show if the primary aim of the TRIAGE study can be fulfilled, but if it is possible to develop a model with the desired sensitivity and specificity of unnecessary admissions, this could be a potential major aid to the physicians in the ED.

There is considerable potential in using biochemichal markers in prognostic staging, also as a tool for identifying low-risk patients [17], and already it has been proven that combinations of vital values and standard blood tests have a high discriminatory power between high and low-risk patients in the acute setting $[24,25]$. However, it has also been shown that the standard blood panel have only limited predictive power in risk assessment when added to physiological parameters [26], future studies can reveal whether prognostic information can be significantly improved using a whole panel of new and old inflammatory biomarkers. Several biomarkers have shown prognostic strength, for example C-reactive protein, suPAR, YKL-40, Pentraxin-3, Copeptin and Troponin T [11-16].

Many patients (44.2\%) in the TRIAGE database experienced no predefined 'events' during admission and hence were not subject to treatment that absolutely necessitated hospitalization. However, some admissions might still have been necessary. Therefore, any patient admission file with no events was scrutinized by medical experts to discover if the admission could be considered necessary. We found a significant relationship between triage category and increasing age, male gender, comorbidities and many predefined events. However, since there were equal amounts of patients with no 'events' in the green, yellow and orange triage categories (Table 3), the DEPT triage model seems inappropriate for predicting the outcome of these patients with lower risk. 
Table 3 Distribution of 'events' according to Triage stage

\begin{tabular}{|c|c|c|c|c|c|}
\hline Panel A - Events qualifying for a necessary admission & Green $(n=1978)$ & Yellow $(n=2386)$ & Orange $(n=1616)$ & $\operatorname{Red}(n=25)$ & $p$-value* \\
\hline No events, n (\%) & $904(45.7)$ & $1087(45.6)$ & $663(41.0)$ & $0(0)$ & $<0.001$ \\
\hline Acute surgery, n (\%) & $51(2.6)$ & $71(3.0)$ & $80(5.0)$ & $4(16)$ & $<0.001$ \\
\hline Surgery during admission, $\mathrm{n}(\%)$ & $125(6.3)$ & $135(5.7)$ & $115(7.1)$ & $2(8)$ & 0.305 \\
\hline Non-scheduled surgery within 14 days, n (\%) & $37(1.8)$ & $46(1.9)$ & $37(2.3)$ & $2(8)$ & 0.145 \\
\hline Trombolysis, n (\%) & $6(0.3)$ & $11(0.5)$ & $11(0.7)$ & $0(0)$ & 0.415 \\
\hline Endovascular procedure, n (\%) & $38(1.9)$ & $50(2.1)$ & $133(8.3)$ & $2(8)$ & $<0.001$ \\
\hline Antibiotics > 24 h within 7 days, $n$ (\%) & $367(18.5)$ & $575(24.1)$ & $284(17.6)$ & $9(36)$ & $<0.001$ \\
\hline i.v.-treatment with diuretics > 1 times within 14 days, n (\%) & $36(1.8)$ & $84(3.5)$ & $73(4.5)$ & $12(48)$ & $<0.001$ \\
\hline Other i.v.-treatment $>1$ times within 14 days, $\mathrm{n}(\%)$ & $414(20.9)$ & $487(20.4)$ & $318(19.7)$ & $14(56)$ & $<0.001$ \\
\hline $\begin{array}{l}\text { Admissions lasting more than } 3 \text { days, not due to social } \\
\text { factors or complications due or to initiated treatments } \\
\text { or investigations within } 30 \text { days, } n(\%)\end{array}$ & $465(23.5)$ & $672(28.2)$ & $513(31.7)$ & $18(72)$ & $<0.001$ \\
\hline Endoscopy detecting GI bleeding within 7 days, n (\%) & $22(1.1)$ & $13(0.5)$ & $6(0.4)$ & $0(0)$ & 0.036 \\
\hline Acute myocardial infarction within 30 days, n (\%) & $1(0.1)$ & $3(0.1)$ & $42(2.6)$ & $0(0)$ & $<0.001$ \\
\hline Ventricular tachycardia within 30 days, $\mathrm{n}(\%)$ & $0(0)$ & $5(0.2)$ & $13(0.8)$ & $1(4)$ & $<0.001$ \\
\hline Cardiac arrest within 30 days, $\mathrm{n}(\%)$ & $5(0.2)$ & $4(0.2)$ & $6(0.4)$ & $1(4)$ & 0.002 \\
\hline Stroke within 30 days, $\mathrm{n}(\%)$ & $11(0.6)$ & $23(1.0)$ & $22(1.4)$ & $2(8)$ & $<0.001$ \\
\hline Transitory Cerebral Ischemia within 30 days, n (\%) & $5(0.2)$ & $14(0.6)$ & $7(0.4)$ & $0(0)$ & 0.406 \\
\hline $\begin{array}{l}\text { Chronic obstructive pulmonary disease requiring non } \\
\text { invasive ventilation within } 7 \text { days, } \mathrm{n}(\%)\end{array}$ & $1(0.1)$ & $12(0.5)$ & $27(1.7)$ & $3(12)$ & $<0.001$ \\
\hline $\begin{array}{l}\text { Transfer to another hospital (apart for rehabilitation) } \\
\text { within the current hospitalization, } \mathrm{n}(\%)\end{array}$ & $62(3.1)$ & $90(3.8)$ & $188(11.6)$ & $7(28)$ & $<0.001$ \\
\hline Admission to intensive care unit within 30 days, $n$ (\%) & $5(0.2)$ & $22(0.9)$ & $47(2.9)$ & $12(48)$ & $<0.001$ \\
\hline $\begin{array}{l}\text { Admission to intermediate-intensive care unit within } 14 \\
\text { days, } \mathrm{n}(\%)\end{array}$ & $6(0.3)$ & $28(1.2)$ & $53(3.3)$ & $6(24)$ & $<0.001$ \\
\hline Death within 30 days, $\mathrm{n}(\%)$ & $44(2.2)$ & $86(3.6)$ & $82(5.1)$ & $7(28)$ & $<0.001$ \\
\hline \multicolumn{6}{|l|}{ Panel B: Events, which identifies a high-risk patient } \\
\hline $\begin{array}{l}\text { Admission to intensive care unit within } 30 \text { days or } \\
\text { semi-intensive care unit within } 14 \text { days, } n(\%)\end{array}$ & $11(0.5)$ & $43(1.8)$ & $90(5.6)$ & $14(56)$ & $<0.001$ \\
\hline Death within 30 days, $\mathrm{n}(\%)$ & $44(2.2)$ & $86(3.6)$ & $82(5.1)$ & $7(28)$ & $<0.001$ \\
\hline
\end{tabular}

${ }^{*} P$ value indicates significant difference between the triage categories. Green/yellow/orange/red refers to triage acuity level in DEPT: non-urgent/urgent/emergent/ resuscitation respectively, (Table 1 for detalis)

\section{Strengths and limitations}

An optimal score in an emergency department should be applicable in all patients as diagnoses might not be clear at patient arrival. However it is possible that the score varies between diagnoses and we have therefore planned subgroup analyses for the different disease categories. Patient inclusion covers many subgroups within internal medicine, surgery and neurology, which is significant strenght giving that the optimal score should be applicable in all patients. The current form of DEPT triage staging has been performed at the hospital for several years and was completely implemented and running steadily thoughout the study period. Earlier medical history was assessed by the patient file and not by questioning, which minimizes reporting bias. We had access to entries in the patient file even after eventual transfer to another hospital. Several investigators participated in extrating the events from the patient files, but each file was assessed by only one investigator and interobserver variability was not adressed. However, the frequency of reporting of events was similar across all investigators except for two events (trombolysis and admission to intensive care) and the differences were very small in absolute numbers, because few patients in the database encountered these two events. This variation is likely coincidence. We are aware that false measurements of vital signs are likely to have occurred as it would in normal clinical practice, but we have not evaluated interobserver agreement between the nurses who performed the measurements.

Nearly $6 \%$ of admissions were not included and this constitutes a selection bias. Furthermore, there were few included patients in the 'red' triage group (0.4\%). 
Therefore, we cannot extrapolate the biomarker levels in the red triage group into a general Danish high-risk population. A comparative analysis shows, that the nonincluded patients were generally more ill. It was evident during data collection, that it was often in the most acute patients that no additional blood sample was taken for the biobank. Additionally; in the capital region of Denmark, seriously injured patients are brought directly to a tertiary trauma centre in Copenhagen, which also means that hyperacute patients are underrepresented in the TRIAGE database. This means our final model will have serious limitations in identifying trauma patients. However importantly, only $3.8 \%$ of the total admitted patients with no 'events' are missing from the TRIAGE database, which probably makes subsequent biomarker levels valid for the general population of low-risk patients admitted to a Danish hospital, keeping in mind that patients with blue triage category (minor complaints and injuries) are not included in the TRIAGE database as they did not fulfill our criteria of admission in the study. Finally, the TRIAGE database is limited since we did not record the precise time the patients spend in the ER before discharge or admission to an inpatient bed. However, these data are not needed to construct the TRIAGE algorithm, since we use 'events' as measurements of outcome. In future studies, when the TRIAGE model could be implemented in the clinic, we have to record ER admission time to conclude if the model has any positive impact on ER crowding as well as the cost effectiveness of the model.

\section{Time schedule and status}

At present time, the database has been completed and further data processing (including the identification of unnecessary hospitalizations) will take place during 2015. Analysis of the biomarkers is on-going and is expected to be finished during 2015 .

\section{Conclusions}

The TRIAGE database has been completed and includes data and blood samples from 6005 unselected consecutive hospitalized patients, corresponding to $94.1 \%$ of all admissions over a three month period. More than $40 \%$ experienced no events and were therefore potentially unnecessary hospital admissions.

\section{Additional files}

Additional file 1: Table S1. Vital values according to Triage stage. (DOCX $120 \mathrm{~kb}$ )

Additional file 2: Table S2. Characteristics of the included and not included admissions. (DOCX 127 kb)

\section{Abbreviations}

ED: Emergency department; LOS: Lenght of stay; COPD: Chronic obstructive pulmonary disease; SUPAR: Soluble urokinase plasminogen activator receptor;
DEPT: Danish emergency process triage; ADAPT: Adaptive process triage; BP: Blood pressure; HR: Heart rate; RR: Respiratory rate; $\mathrm{SpO}_{2}$ : Saturation of peripheral oxygen; Tp: Temperature; SD: Standard deviation; Cl: Confidence interval.

\section{Competing interests}

The authors have nothing to declare.

\section{Authors' contributions}

$\mathrm{Kl}$ is the primary initiator of the TRIAGE study, wrote the first draft of the protocol and instructed the emergency department prior to the study. LLP wrote the manuscript, performed the statistical analyses and participated in data collection. ASI, SL, TLN, RØ, PEW, IAS, MK participated in the collection of data and all critically revised the script. JLF, LK, LSR, GS, BKP, JEO and KI initated the TRIAGE study, participated with interpretation of the results of the present study and all critically revised the script. All authors read and approved the final version of the article.

\section{Authors' information}

Authors academic titles: Kasper Iversen MD DMSc, Jakob Lundager Forberg MD, Lars Køber MD Professor DMSc, Lars S. Rasmussen MD Professor DMSc, György Sölétormos MD DMSc, Bente Klarlund Pedersen MD Professor DMSc, Jesper Eugen-Olsen ${ }^{3}$ Ph.D, Michael Kristensen MD. Louis Lind Plesner, Anne Kristine Servais Iversen, Sandra Langkjær, Ture Lange Nielsen, Rebecca Østervig, Peder Emil Warming and Idrees Ahmad Salam are all medical students.

\section{Acknowledgements}

LSR has received funding from the Tryg foundation.

\section{Author details}

'Department of Cardiology, Endocrinology and Nephrology, North Zealand Hospital, Copenhagen University Hospital, Copenhagen, Denmark. ${ }^{2}$ Department of Cardiology, Herlev Hospital, Copenhagen University Hospital, Copenhagen, Denmark. ${ }^{3}$ Clinical Research Centre, Copenhagen University Hospital Hvidovre, Copenhagen, Denmark. ${ }^{4}$ Emergency Department, North Zealand Hospital, Copenhagen University Hospital, Copenhagen, Denmark. ${ }^{5}$ Department of Cardiology, Rigshospitalet, Copenhagen University Hospital, Copenhagen, Denmark. ${ }^{6}$ Department of Anaesthesia, Centre of Head and Orthopaedics, Rigshospitalet, University of Copenhagen, Copenhagen, Denmark. ${ }^{7}$ Department of Clinical Biochemistry, North Zealand Hospital, Copenhagen University Hospital, Copenhagen, Denmark. ${ }^{8}$ Centre of Inflammation and Metabolism (CIM) and Centre for Physical Activity Research (CFAS), Rigshospitalet, Copenhagen University Hospital, Copenhagen, Denmark.

Received: 30 June 2015 Accepted: 12 November 2015

Published online: 01 December 2015

\section{References}

1. Statistics Denmark [www.statbank.dk]. Accessed June 12, 2015.

2. Carter EJ, Pouch SM, Larson EL. The relationship between emergency department crowding and patient outcomes: A systematic review. J Nurs Sch. 2014:46:106-15.

3. Sprivulis PC, Da Silva JA, Jacobs IG, Frazer AR, Jelinek GA. The association between hospital overcrowding and mortality among patients admitted via Western Australian emergency departments. Med J Aust. 2006;184:208-12.

4. Madsen F, Ladelund S, Linneberg A. High levels of bed occupancy associated with increased inpatient and thirty-day hospital mortality in Denmark. Health Aff (Millwood). 2014;33:1236-44.

5. O'Cathain A, Knowles E, Maheswaran R, Pearson T, Turner J, Hirst E, et al. A system-wide approach to explaining variation in potentially avoidable emergency admissions- national ecological study. BMJ Qual Saf. 2014;23:47-55.

6. Trentino KM, Swain SG, Burrows SA, Sprivulis PC, Daly FFS. Measuring the incidence of hospital-acquired complications and their effect on length of stay using CHADx. Med J Aust. 2013;199:543-7.

7. Basse L, Thorbø| JE, Løssl K, Kehlet H. Colonic surgery with accelerated rehabilitation or conventional care. Dis Colon Rectum. 2004;47:271-7. 277-8.

8. Shewale JB, Correa AM, Baker CM, Villafane-Ferriol N, Hofstetter WL, Jordan VS, et al. Impact of a fast-track esophagectomy protocol on esophageal cancer patient outcomes and hospital charges. Ann Surg. 2014;00:1-10.

9. Robertson-Steel I. Evolution of triage systems. Emerg Med J. 2006;23:154-5 
10. Segal M: Dual Eligible Beneficiaries and Potentially Avoidable Hospitalizations. 2011.

11. Bastrup-Birk S, Munthe-Fog L, Skjoedt M-O, Ma YJ, Nielsen H, Køber L, et al. Pentraxin-3 level at admission is a strong predictor of short-term mortality in a community-based hospital setting. J Intern Med. 2014;3:1-11.

12. Kaptoge S, Di Angelantonio E, Lowe G, Pepys M, Thompson S. C-reactive protein concentration and risk of coronary heart disease, stroke, and mortality: an individual participant meta- analysis. Lancet. 2014;375:132-40.

13. Lyngbæk S, Andersson C, Marott JL, Møller DV, Christiansen M, Iversen KK, et al. Soluble urokinase plasminogen activator receptor for risk prediction in patients admitted with acute chest pain. Clin Chem. 2013;59:1621-9.

14. Iversen K, Køber L, Gøtze JP, Dalsgaard M, Nielsen H, Boesgaard S, et al. Troponin $\mathrm{T}$ is a strong marker of mortality in hospitalized patients. Int J Cardiol. 2013;168:818-24

15. Mygind ND, Iversen K, Køber L, Goetze JP, Nielsen H, Boesgaard S, et al. The inflammatory biomarker YKL-40 at admission is a strong predictor of overall mortality. J Intern Med. 2013;273:205-16.

16. Iversen K, Gøtze JP, Dalsgaard M, Nielsen H, Boesgaard S, Bay M, et al. Risk stratification in emergency patients by copeptin. BMC Med. 2014;12:80.

17. Seymour CW, Cooke CR, Wang Z, Kerr KF, Ph D, Yealy DM, et al. Improving risk classification of critical illness with biomarkers: a simulation study. J Crit Care. 2014:28:541-8.

18. North Zealand Hospital Webpage [http://www.nordsjaellandshospital.dk] Accessed October 20, 2014

19. Danish emergency process triage [http://www.deptriage.dk]. Accessed November 11, 2014

20. Nordberg, M. Lethvall, S. Castren M: The validity of the triage system ADAPT. Scand J Trauma Resusc Emerg Med. 2010;18(Suppl 1):P36.

21. Institute CLS. Procedures for collection of diagnostic blood specimens by venipuncture; approved guideline, 6th ed. CLSI document H3-A6. Wayne, PA: CLSI; 2007.

22. Gertman PM, Restuccia JD. The appropriateness evaluation protocol: a technique for assessing unnecessary days of hospital care. Med Care. 1981; 19:855-71.

23. Lemeshow S, Klar J, Teres D. Outcome prediction for individual intensive care patients: useful, misused, or abused? Intensive Care Med. 1995;21:770-6.

24. Kellett J, Deane B. The simple clinical score predicts mortality for 30 days after admission to an acute medical unit. QJM. 2006;99:771-81.

25. Silke B, Kellett J, Rooney T, Bennett K, O'Riordan D. An improved medical admissions risk system using multivariable fractional polynomial logistic regression modelling. QJM. 2010;103:23-32.

26. Goodacre S, Wilson R, Shephard N, Nicholl J. Derivation and validation of a risk adjustment model for predicting seven day mortality in emergency medical admissions: mixed prospective and retrospective cohort study. BMJ. 2012;344, e2904

\section{Submit your next manuscript to BioMed Central and take full advantage of:}

- Convenient online submission

- Thorough peer review

- No space constraints or color figure charges

- Immediate publication on acceptance

- Inclusion in PubMed, CAS, Scopus and Google Scholar

- Research which is freely available for redistribution 